\title{
METHOD FOR EVALUATING THE PROGRESSIVE COLLAPSE RESISTANCE OF LONG-SPAN SINGLE-LAYER SPATIAL GRID STRUCTURES
}

\author{
Li-Min Tian ${ }^{1,}{ }^{*}$, Jian-Peng Wei ${ }^{1}$ and Ji-Ping Hao ${ }^{1}$ \\ ${ }^{1}$ School of Civil Engineering, Xi'an University of Architecture and Technology, Xi'an, China \\ * (Corresponding author: E-mail: tianlimin@xauat.edu.cn)
}

\section{A B S T RA C T}

In recent years, researchers have increasingly reported the progressive collapse of long-span spatial grid structures. However, research on long-span spatial grid structures, especially single-layer spatial grid structures, remains limited. Thus, this study conducted an incremental dynamic analysis to develop a suitable method for evaluating single-layer spatial grid structures. The method uses a quantitative evaluation index called the collapse margin ratio. Engineering cases were analysed to validate the proposed method. For instance, the proposed method was used to evaluate the progressive collapse of the main stadium of the Shenzhen Universiade Sports Centre. The results showed that the roof of the structure has good resistance against progressive collapse from the perspective of quantitative analysis, even though the cantilever length reached 51.9-68.4 m at some locations, and the novel method is not restricted by the structural form. Thus, the method can be used to quantitatively evaluate a structure's resistance to collapse.

\section{A R T I C LE H I S T O R Y}

$\begin{array}{ll}\text { Received: } & \text { 31 August } 2017 \\ \text { Revised: } & \text { 21 February } 2018 \\ \text { Accepted: } & \text { 28 March } 2018\end{array}$

\section{K E Y W O R D S}

Single-layer spatial grid

structure;

Progressive collapse;

Evaluation method;

Incremental dynamic analysis;

Vulnerability curve;

Collapse margin ratio

\section{Introduction}

Progressive collapse is defined as the spread of an initial local failure from element to element, which eventually results in the collapse of the entire structure or a disproportionately large part of it $[1,2]$. Since the collapse of the Romexpo Pavilion in Bucharest, Romania ( which was comprised of a singlelayer spherical reticulated shell with a span of $93.5 \mathrm{~m}$ and rise of $19.1 \mathrm{~m}$ ) in 1961 and the destruction of the Hartford Civic Centre ( which was comprised of a space truss structure with a span of $110 \mathrm{~m}$ ) in 1978, the traditional concept that the progressive collapse of a spatial grid structure following the loss of one important member can be prevented by a large degree of redundancy has been changing $[3,4]$. Cases about the progressive collapse of long-span spatial grid structures have been reported more frequently than ever because of the widespread use of such structures in public buildings, which has enhanced socioeconomic development $[5,6,7]$.

Studies regarding the progressive collapse have mostly focused on the frame structure $[8,9,10]$. Researchers have conducted several progressive collapse experiments for a single-column removal scenario [11] and have compared various modelling approaches for steel frames [12]. Moreover, engineers have developed design specifications for steel frames for the prevention of progressive collapse $[13,14,15]$. However, research on long-span spatial grid structures, especially single-layer spatial grid structures, has been rather limited [16, 17, 18]. A new beam-column element was presented [19] and adopted in a second-order direct analysis of a long-span single-layer roof [20]. The collapse of a single-layer spatial grid structure, which occurs due to the accumulation of node failures and member buckling, is different from that of a frame, wherein a sufficiently plastic hinge is formed in the structure and induces progressive collapse. The load-carrying mechanism of a single-layer spatial grid structure relies heavily on its structural shape. Therefore, evaluation methods that are appropriate for frames cannot be used directly for single-layer spatial grid structures [21]. Thus, a new method must be developed to evaluate single-layer spatial grid structures specifically.

Incremental dynamic analysis (IDA) considers several types of random factors to evaluate the structural seismic performance without being limited by the structural form [22]. The progressive collapse induced by member removal is different from that in the case of an earthquake. Based on the principle of IDA, this study developed a suitable method for evaluating single-layer spatial grid structures. The seismic waves were replaced by the failure of a different member in order to conduct a series of dynamic and time-history analyses. Moreover, vulnerability analysis was conducted to describe the probability of the exceedance of collapse states. A quantitative evaluation index called the collapse margin ratio (CMR) was derived. The proposed method was validated by analysing several engineering cases, and the influence of an initial geometric imperfection was analysed. The evaluation method was then utilised in a computer simulation of the main stadium for the Shenzhen Universiade Sports Centre and was found to provide accurate and reliable results for the progressive collapse of a long-span single-layer spatial grid structure. The finite element
(FE) analysis performed in this study was validated by conducting an experiment on the progressive collapse of a single-layer latticed dome [4].

\section{Evaluation method based on an incremental dynamic analysis}

\subsection{Basic principle of evaluation method}

IDA can be regarded as an improvement over static pushover analysis, wherein the ground motion intensity measure (IM) is continuously increased to obtain the corresponding damage measure (DM). A curve is plotted with the DM and IM along the horizontal and vertical axes, respectively. Multiple DMIM curves can be drawn for various seismic waves to evaluate the ability of a structure to resist collapse.

Although the specific causes of collapse differ in the case of an earthquake and the loss of an important member, they are similar in that both are dynamic processes that occur due to an accidental event. Thus, the principle of IDA can be used for evaluating single-layer spatial grid structures. Unlike a seismic wave, the failure of a different member is considered a cause of structural vibration. To consider the influence of various loads, the combined load is increased such that the intensity is changed until the progressive collapse of the structure occurs. The potential ability of a single-layer spatial grid structure to resist progressive collapse and its performance for various combined loads can be obtained, and its resistance to progressive collapse can be quantitatively evaluated.

\subsection{Evaluation procedure}

A suitable method for evaluating single-layer spatial grid structures was developed based on the principle of IDA. The main steps are given below.

(1) A certain number of important members are selected through a multipleresponse evaluation method based on the primary scope [7]. The maximum displacement that directly produces a damaged condition for the structure is chosen as the DM, and the combined load that reflects the vibration strength is chosen as the IM. These are used to derive a load-displacement curve in a manner that is similar to a pushdown analysis.

(2) The loss of one important member is considered. An alternate load path method along with the consideration of the construction effect [7] is used to obtain the DM when the IM is increased or decreased through a change in the live load without changing the dead load. This may cause the DM to increase significantly with a slight increment in the IM. The DM-IM curve is then plotted. It should be noted that the load increment should be reduced in order to capture the change in the DM-IM curve more clearly during the plastic development process. The two reasons why only the single-member failure condition was considered in this study are that member failure is a smallprobability event, and the removal of a single member can reflect the ability to form other load paths.

(3) Step (2) is repeated using different important members to obtain more DM-IM curves.

(4) The DM-IM curves are processed according to the evaluation standard 
to evaluate the resistance of the structure to progressive collapse.

The method is called evaluation of progressive-collapse resistance based on IDA, and the basic process is presented in Fig. 1. This method can be regarded as a series of dynamic and time-history analyses in which the alternate load path method along with the consideration of the construction effect are applied.

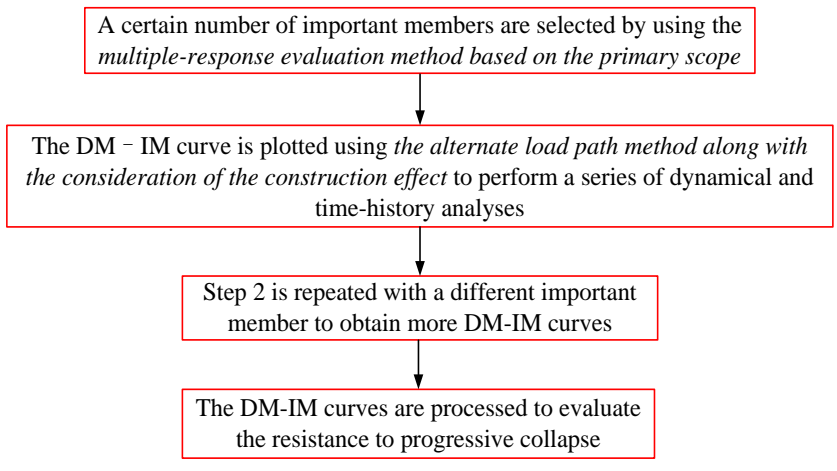

Fig. 1 Basic procedure of the evaluation method

\subsection{Evaluation standard}

To demonstrate the structural performance of anti-collapse, a statistical method is required to address the discrete DM-IM curves. The $16 \%, 50 \%$, and $84 \%$ quantile curves for qualitative analysis can be obtained to characterise the average level and discreteness of the curves [23]. The specific procedure is as follows (Fig. 2):

(1) The fitting method of a spline or polynomial curve can be imposed on discrete data points to obtain DM-IM curves. Regardless of the fitting method used, the fitting result should be precisely consistent with the DM-IM curves.

(2) DM-IM curves can be summarised using parametric or nonparametric methods. The former assumes that each DM-IM curve obeys an exponential distribution. Regression analysis is used to obtain the exponential model parameters for plotting the DM-IM curve. For the latter, the mean value and logarithmic standard deviation of the IM are calculated at certain DMs to obtain the curves of (DM, $\left.\mu e^{-\delta}\right),(\mathrm{DM}, \mu)$, and (DM, $\left.\mu e^{+\delta}\right)$, which represent the $16 \%$, $50 \%$, and $84 \%$ quantile curves, respectively. The calculation method is the same as that used for the various IMs.

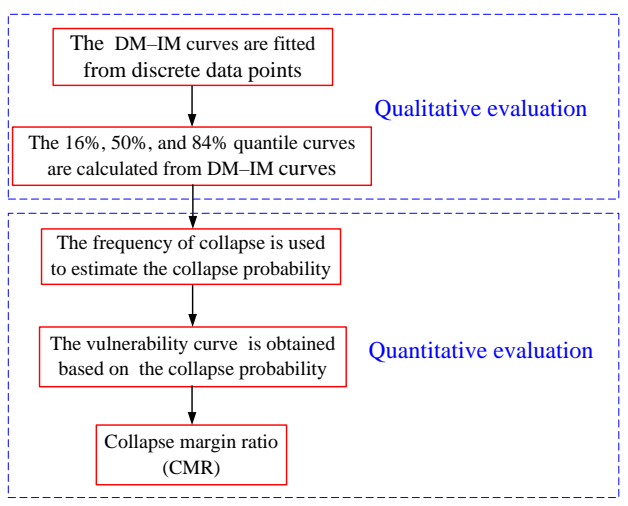

Fig. 2 Evaluation standard

A quantitative analysis is also necessary. A structural vulnerability curve that reflects the probability of progressive collapse for accidents with various intensities can be depicted using the IM parameters for the collapse point of each DM-IM curve $[24,25]$. The CMR of the structure can then be calculated based on the vulnerability curve. The specific procedure is as follows (Fig. 2):

(1) The frequency of collapse is used to estimate the collapse probability for different IMs:

$P[\mathrm{C} \mid \mathrm{IM}=i m]=N_{\mathrm{C}} / N$

where $P[\mathrm{C} \mid \mathrm{IM}=\mathrm{im}]$ is the collapse probability of the structure when $\mathrm{IM}=i m$, and $N$ is the number of important members for which $N_{\mathrm{C}}$ important members cause the progressive collapse of the structure.

(2) A logarithmic normal distribution model is adopted for the vulnerability function, and the vulnerability curve in Eq. 2 can be fitted to the collapse probability of each IM:

$P[\mathrm{C} \mid \mathrm{IM}=i m]=\Phi[\ln (\mathrm{im} / \mathrm{m}) / \beta]$

where $m$ and $\beta$ are the mean value and logarithmic standard deviation, respectively, of the structure's ability to resist progressive collapse.

(3) $\mathrm{IM}_{50 \%}$ corresponds to a $50 \%$ collapse in probability and was adopted as the resistance index of progressive collapse. The accidental events were the main reason for a progressive collapse, which has a lower probability of occurring. The dead load was in a dominant position, and thus, the live load was reduced, and the wind load was ignored. Consequently, $\mathrm{IM}_{\mathrm{o}}$ was set to 1.2 times the dead load and 0.5 times the live load (1.2DL+0.5LL, [13, 14]). The combined load is used to determine the ability of a structure to resist collapse. The ratio of $\mathrm{IM}_{50 \%}$ to $\mathrm{IM}_{\mathrm{o}}$ is the CMR:

$\mathrm{CMR}=\mathrm{IM}_{50 \%} / \mathrm{IM}_{\mathrm{o}}$

\subsection{Collapse standard}

Based on the characteristics of a single-layer spatial grid structure, its progressive collapse after partial destruction occurs due to the accumulation of node failures and member buckling, which diminish the overall stiffness of the structure. Therefore, the collapse point in a DM-IM curve is defined as the point at which the overall stiffness is reduced to $20 \%$ of $K_{\mathrm{e}}$, where $K_{\mathrm{e}}$ is the initial slope of the curve and indicates the original stiffness of the structure [26]. It should be noted that a single-layer spatial grid structure with few members may collapse without a decrease in stiffness. In this situation, the actual collapse point is adopted.

\section{Verification of finite element analysis}

\subsection{Analysis model}

To validate the FE analysis performed in this study, a single-layer latticed dome was tested, as shown in Fig. 3. The span and rise were 4.2 and $0.7 \mathrm{~m}$, respectively, and the meridians were equally divided on the horizontal projection plane. In addition, the edge supports were set as fixed ideal pins to make this experimental program a benchmark for the validation of the FE analysis. A two-node space beam element [27] was employed for each member, and the members were connected by rigid joints. To accurately simulate the collapse process, material and geometric nonlinearity were considered. This modelling approach was adopted for the subsequent case studies and engineering applications. Further details regarding this test model are provided by Zhao et al. [4].

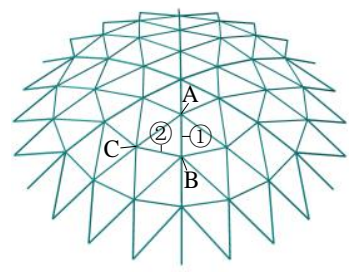

(a) Axonometric drawing
Fig. 3 Test model

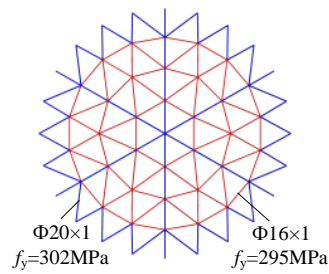

(b) Plane drawing
The test model was subjected to a point load of $0.8 \mathrm{kN}$ using weights. For a beam element with virtual density, the mass centre and stiffness centre were coincided with the linked joint to avoid the application of torsion at each joint, as shown in Fig. 4. Member 1 was eliminated by a member-breaking device after static loading. Fig. 5 presents the decreased ratio of the axial force in member 1 ; the axial force decreased quickly and was reduced to zero in $0.26 \mathrm{~s}$ Therefore, the axial force of member 1 in the FE analysis was plotted along the curve shown in Fig. 5 until it reached zero.

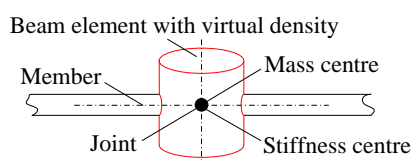

Fig. 4 Beam element with virtual density

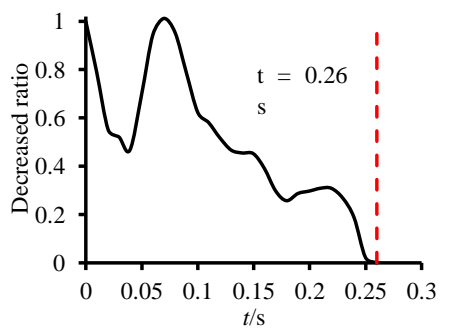

Fig. 5 Decreased ratio of axial force in member 1 
First, an implicit time integration analysis (Newton-Raphson iteration algorithm) was performed to obtain the initial static condition of the intact dome. Second, the deformed mesh and material state of the dome without member 1 were imported into the explicit time integration solver (central difference method) for the dynamic analysis of progressive collapse. A damping ratio of 0.02 [28] was considered as the Rayleigh damping, but the stiffness damping was ignored in the explicit time integration solver to eliminate the requirement for calculating a stiffness matrix and to reduce the computational costs.

\subsection{Results}

Fig. 6 presents the displacements of joints near member 1 and the strain of member 2 near joint B. A good agreement was observed between the FE and test results; the values for the maximum displacement and strain matched well. In addition, the balance state was regained after member 1 was eliminated, which matched the test observation. Therefore, it was verified that the FE analysis used in this study provides efficient and accurate results.

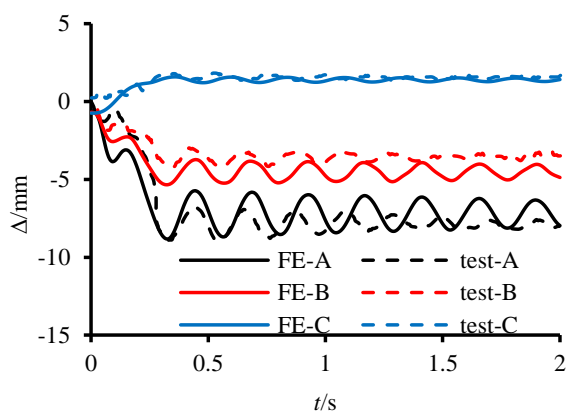

(a) Vertical displacement

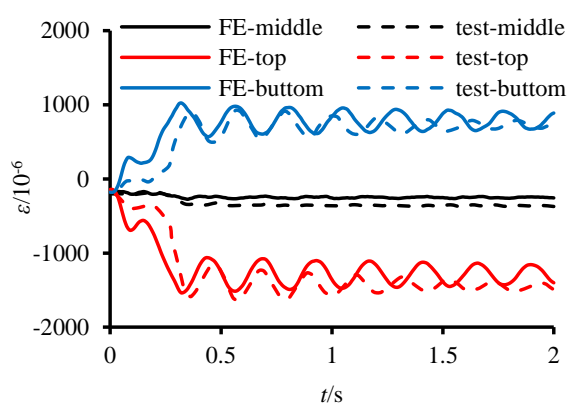

(b) Strain of member 2 near joint $B$

Fig. 6 Comparison between FE and test results

Fig. 7 shows the initial state with a point load of $0.8 \mathrm{kN}$. The maximum stress was $42.3 \mathrm{MPa}$, and the displacement was negligible. Fig. 8 plots the distributions of the stress and displacement when member 1 was eliminated and the peak values were reached. The maximum values were concentrated in the local area next to joint $\mathrm{B}$, which showed considerable resistance to an unbalanced load. The maximum displacement remained low, and the stress was less than the yield stress of the dome. Thus, progressive collapse was prevented.

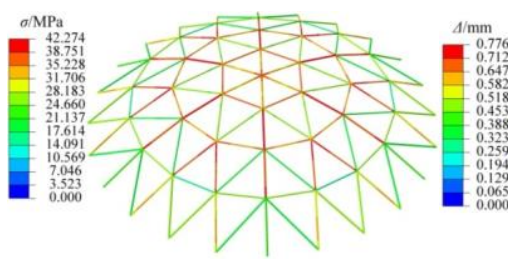

(a) Stress distribution

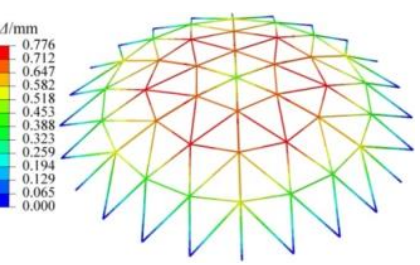

(b) Displacement distribution
Fig. 7 Initial state

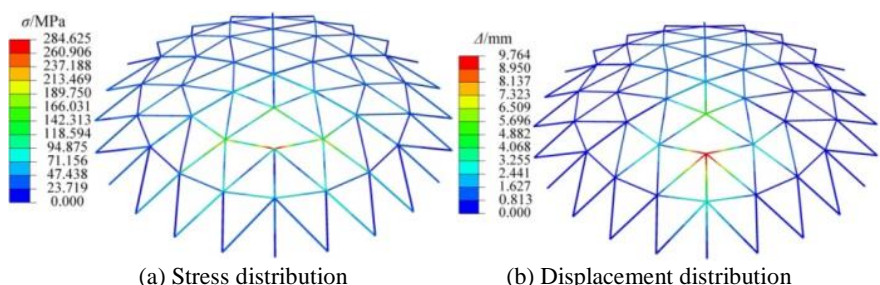

Fig. 8 Member 1 is eliminated

\section{Verification of the evaluation method}

\subsection{Engineering Cases}

To validate the evaluation method, three typical single-layer spatial grid structures [28] representing latticed shells with nonnegative, zero, and negative Gaussian curvatures were analysed. The specific arrangements of the members and structural geometric parameters are shown in Fig. 9. The boundary conditions were determined based on the practical conditions of the structures. The surrounding nodes of the spherical latticed shell were considered as solid joints. For the cylindrical latticed shell, fixed hinge supports were used on two longitudinal edges, and the rest of the edges only restricted the in-plane displacement. For the hyperbolic paraboloid latticed shell, the vertical displacement of the edge nodes was limited except in the case of four corner nodes with fixed hinge supports.

A circular steel pipe was used in the three single-layer spatial grid structures. The specific member sizes are listed in Table 1. A greater stiffness was required for the boundary members of the hyperbolic paraboloid latticed shell than for the rest of members. A cross-section of $\Phi 630 \mathrm{~mm} \times 16 \mathrm{~mm}$ was adopted, which is approximately eight times larger than that of the chord members. The members were made of the material Q235 with a standard yield strength of 235 $\mathrm{MPa}$, and a bilinear isotropic constitutive model that considers the material reinforcement was selected to examine the dynamic collapse process. The member weights were automatically calculated using the FE software, and the node weights were estimated to have $25 \%$ of the member weights imposed. The roof material $\left(1.5 \mathrm{kN} / \mathrm{m}^{2}\right)$ and live load $\left(0.5 \mathrm{kN} / \mathrm{m}^{2}\right)$ were applied to the nodes of the shells using an equivalent calculation.

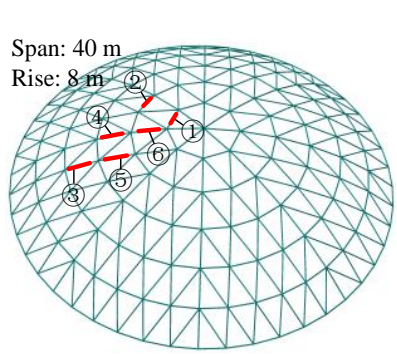

(a) Spherical latticed shell

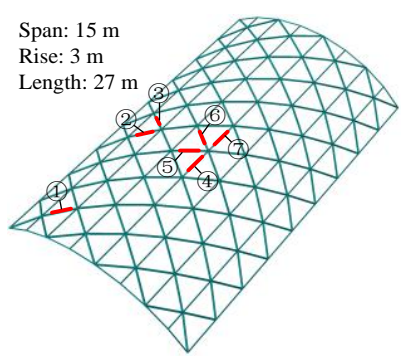

(b) Cylindrical latticed shell

Fig. 9 Three typical single-layer spatial grid structures

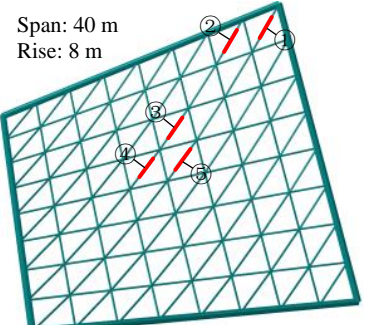

(c) Hyperbolic paraboloid latticed shel
Table 1

Member sizes

\begin{tabular}{|c|c|c|}
\hline Structural form & Member location & Cross-section \\
\hline \multirow{2}{*}{ Spherical latticed shell } & Meridian and parallel member & $\Phi 121 \times 3.5$ \\
\cline { 2 - 3 } & Diagonal member & $\Phi 114 \times 3$ \\
\hline \multirow{2}{*}{$\begin{array}{c}\text { Cylindrical latticed shell } \\
\text { Hyperbolic paraboloid latticed } \\
\text { shell }\end{array}$} & Longitudinal and short edge member & $\Phi 89 \times 4$ \\
\cline { 2 - 3 } & Diagonal member & $\Phi 140 \times 6$ \\
\cline { 2 - 3 } & Chord member & $\Phi 180 \times 7$ \\
\hline
\end{tabular}

\subsection{Quantile curves analysis}

The multiple-response evaluation method based on the primary scope [7] was applied to the three single-layer spatial grid structures in order to determine the distribution of the important members, as shown in Fig. 9. The resistance to progressive collapse was evaluated using IDA. Fig. 10 shows the progressive collapse of the three single-layer spatial grid structures when member 1 was eliminated and the ultimate load condition was achieved. The structures as well as other important members lost bearing capacity and collapsed completely in $3 \mathrm{~s}$. 

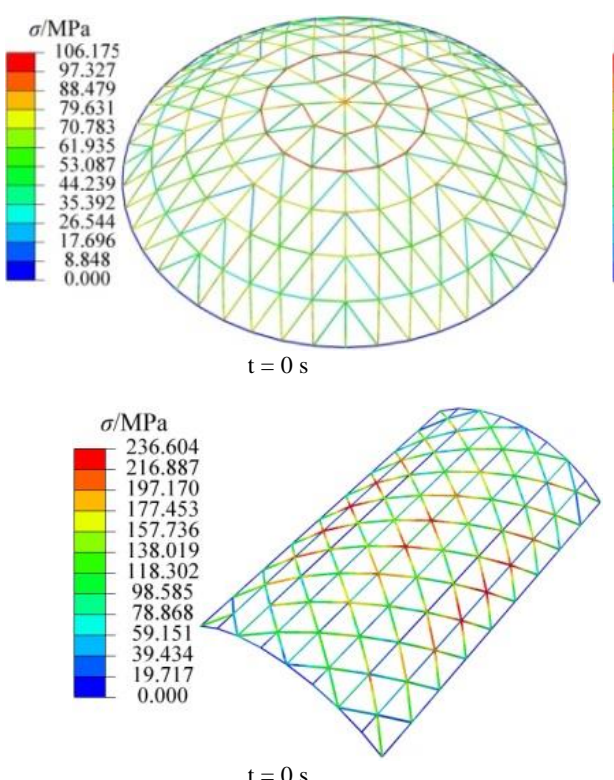

$t=0 s$

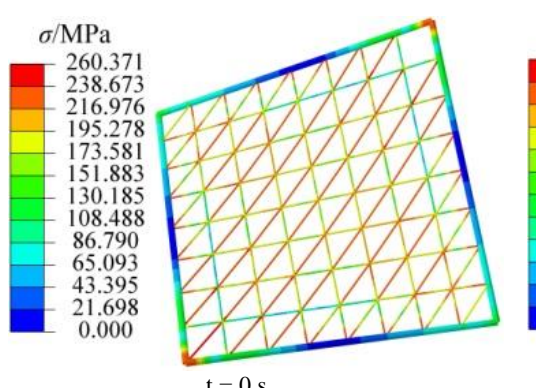

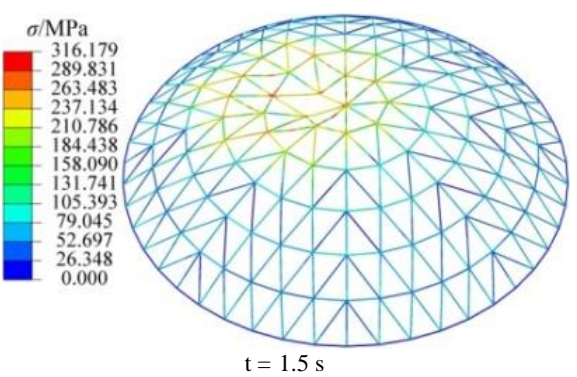

(a) Spherical latticed shell

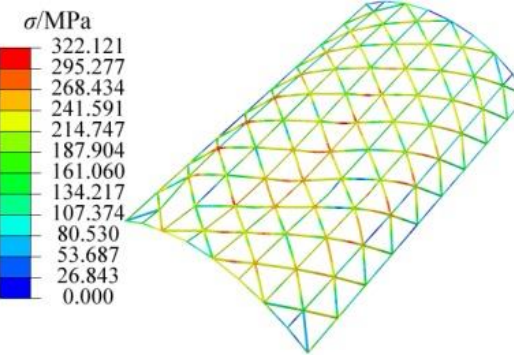

$\mathrm{t}=0.8 \mathrm{~s}$

(b) Cylindrical latticed shell

$\sigma / \mathrm{MPa}$

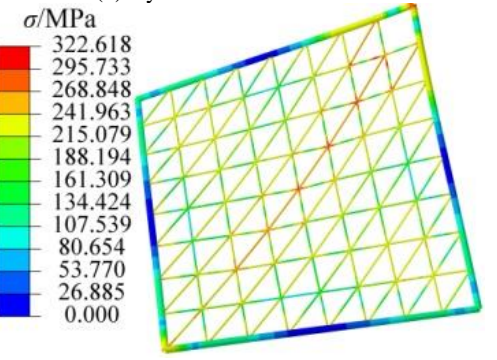

$\mathrm{t}=1.0 \mathrm{~s}$
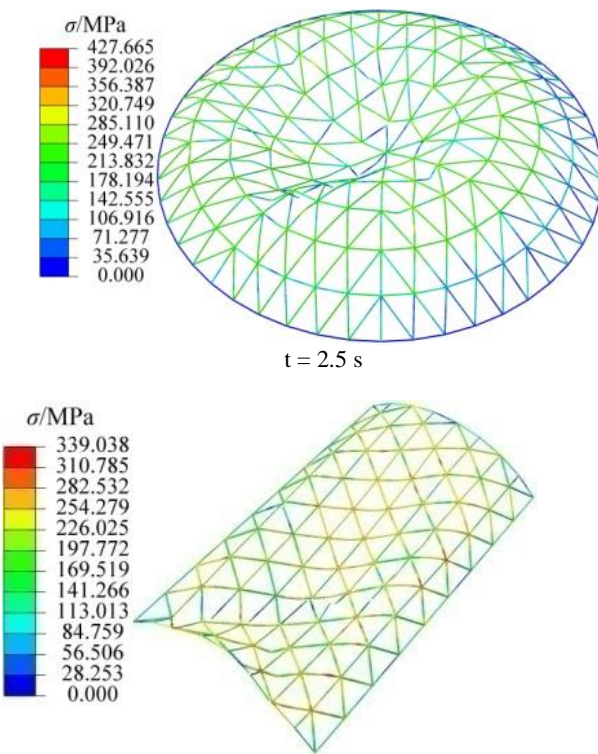

$\mathrm{t}=1.0 \mathrm{~s}$

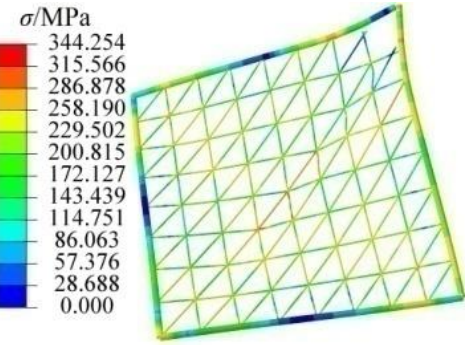

$\mathrm{t}=1.6 \mathrm{~s}$

(c) Hyperbolic paraboloid latticed shell

Fig. 10 Progressive collapse of structures

Fig. 11 presents the DM-IM curves of the original model after each important member failure. The partial coefficient of the live load $\gamma$ was chosen as the vertical axis because the partial coefficient of the dead load was fixed at 1.2 [13, 14]. Fig. 12 presents the DM-IM curves when an initial geometric imperfection was considered based on the consistent mode imperfection method The imperfection distribution was the same as the first-order buckling mode under a vertical load. The magnitude of the initial imperfection was $1 / 300$, where 1 is the span of the structure [28]. Therefore, the initial imperfections were set as $50 \mathrm{~mm}$ for the cylindrical latticed shell and $133 \mathrm{~mm}$ for the other two latticed shells. The nonparametric method was used to calculate the $16 \%, 50 \%$, and $84 \%$ quantile curves for each DM, as shown in Figs. 13 and 14

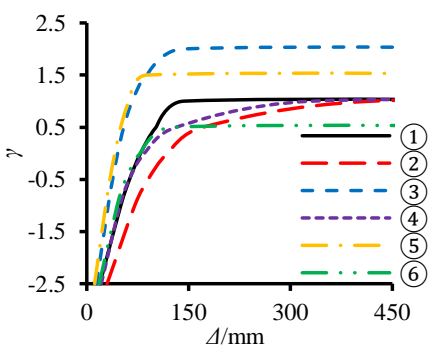

(a) Spherical latticed shell

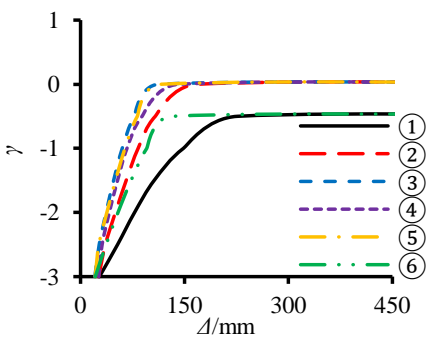

(a) Spherical latticed shell

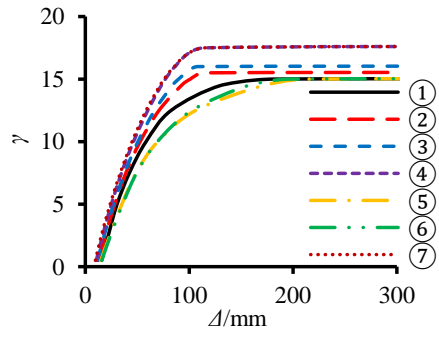

(b) Cylindrical latticed shell

Fig. 11 DM-IM curves of the original model

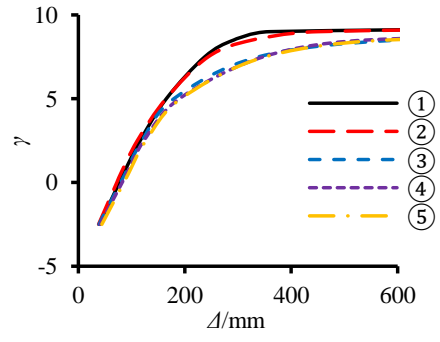

(c) Hyperbolic paraboloid latticed shell

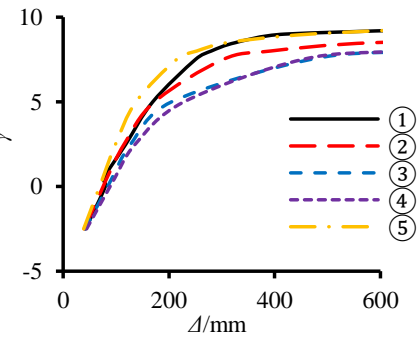

(c) Hyperbolic paraboloid latticed shell

Fig. 12 DM-IM curves considering the initial geometric imperfection 


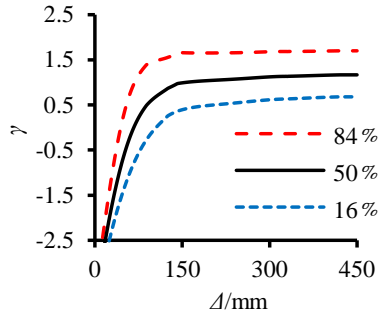

(a) Spherical latticed shell

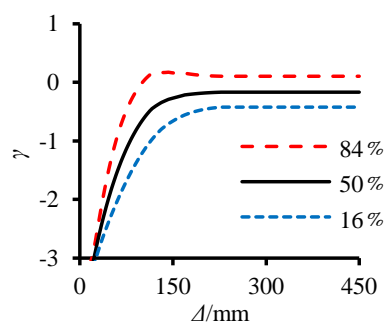

(a) Spherical latticed shell

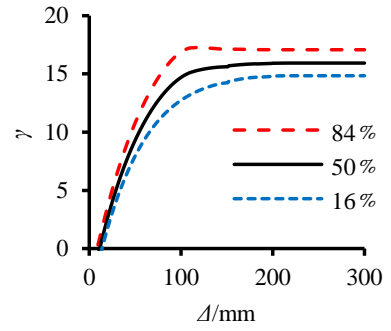

(b) Cylindrical latticed shell

Fig. 13 Quantile curves of the original model

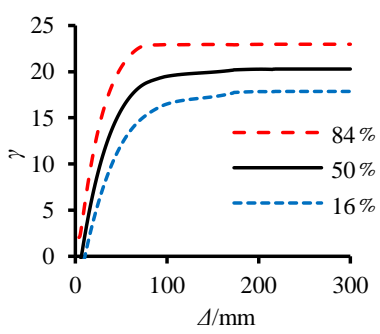

(b) Cylindrical latticed shell

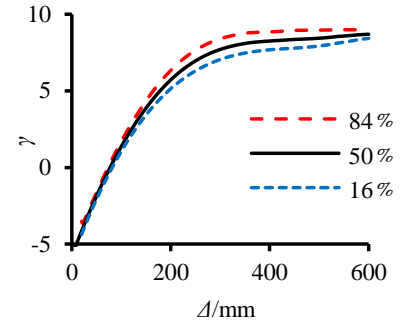

(c) Hyperbolic paraboloid latticed shell

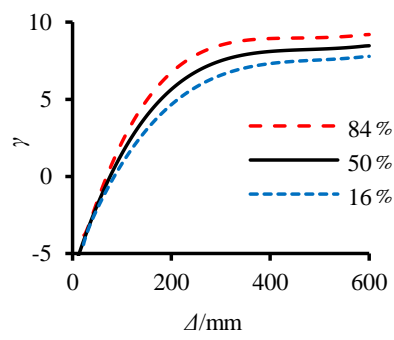

(c) Hyperbolic paraboloid latticed shell

Fig. 14 Quantile curves considering the initial geometric imperfection

The analysis showed that the performance level of the structure can be divided into two stages. First, the slope of the curve remains nearly constant in the initial stage, wherein the entire structure stays in an elastic state as the members deform elastically. Second, as members yield or buckle with an increasing live load, the slope of the curve clearly decreases until the ultimate load condition is finally reached. Therefore, the collapse point is obtained when the slope of the curve is reduced to $20 \%$ of $K_{\mathrm{e}}$.

In the case of the spherical and cylindrical latticed shells, the effects of the initial geometric imperfection were more serious than for the hyperbolic paraboloid latticed shell. This is because the majority of the members experienced compression. Based on the obtained results, the bearing capacity of the spherical latticed shell decreased when the initial geometric imperfection was considered, while the bearing capacity of the cylindrical latticed shell increased considerably due to shape distortion.

The quantile curves revealed the discreteness of the results. Thus, the difference in the importance of the members can be recognised, and the weak links of a structure can be identified. After the initial geometric imperfection was imposed, the discreteness of the results for the cylindrical and hyperbolic paraboloid latticed shells increased. This indicates that considering an initial geometric imperfection increases the difference in importance of the members and highlights weak links.

\subsection{Vulnerability curves analysis}

These curves only allow for a qualitative analysis of collapse resistance. The cylindrical latticed shell showed the best resistance to progressive collapse among the structures in terms of bearing capacity and was followed by the hyperbolic paraboloid latticed shell. The spherical latticed shell could not even bear the dead load. Hence, a quantitative analysis is proposed to calculate the relationship between the collapse probability and IM parameters, which is shown in Figs. 15 and 16. The CMR can then be obtained based on the vulnerability curves, whose results are listed in Table 2.

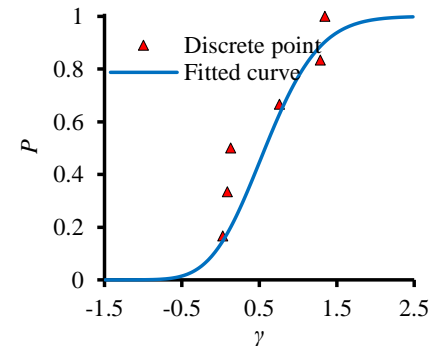

(a) Spherical latticed shell

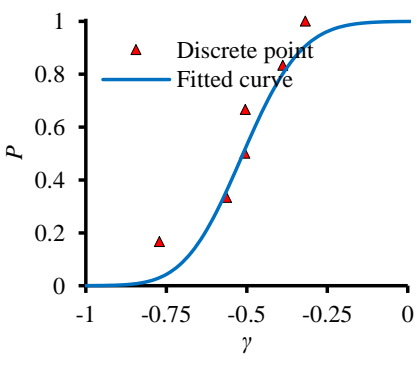

(a) Spherical latticed shell

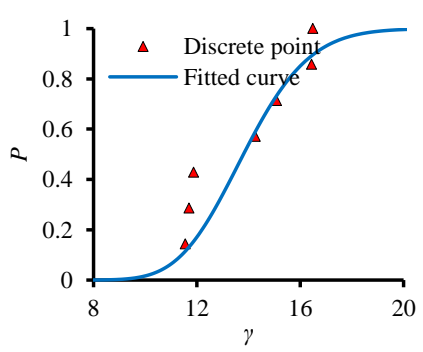

(b) Cylindrical latticed shell Fig. 15 Vulnerability curves of the original model

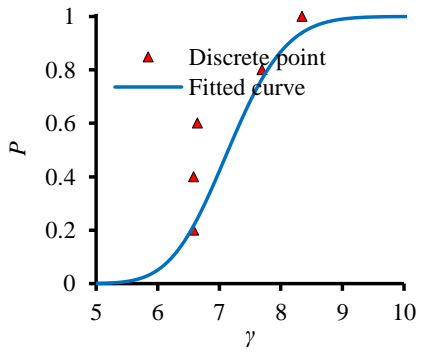

(c) Hyperbolic paraboloid latticed shell (b) Cylindrical latticed shell

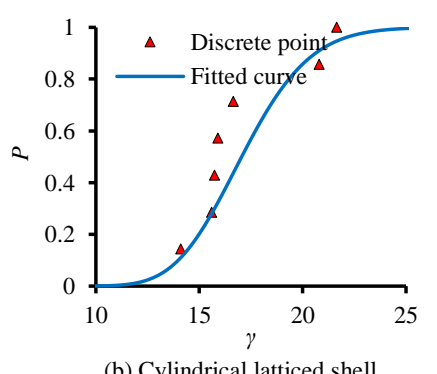

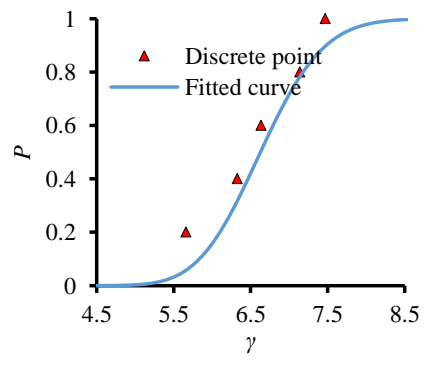

(c) Hyperbolic paraboloid latticed shell
Table 2

Results of CMR

\begin{tabular}{|c|c|c|c|}
\hline Structural form & $\begin{array}{c}\text { Spherical } \\
\text { latticed shell }\end{array}$ & $\begin{array}{c}\text { Cylindrical } \\
\text { latticed shell }\end{array}$ & $\begin{array}{c}\text { Hyperbolic } \\
\text { paraboloid latticed } \\
\text { shell }\end{array}$ \\
\hline Original model & 1.01 & 3.55 & 2.27 \\
\hline $\begin{array}{c}\text { Initial geometric } \\
\text { imperfection } \\
\text { considered }\end{array}$ & 0.80 & 4.81 & 2.17 \\
\hline
\end{tabular}

The vulnerability curves indicated that the resistance to progressive collapse can be described comprehensively, and the corresponding collapse probability can be determined for various combined loads. As compared to the cylindrical and hyperbolic paraboloid latticed shells, the collapse points of the spherical latticed shell were more concentrated. This illustrates that the differences in mechanical performance of the important members were small 
and that the overall structure was uniformly forced.

Table 1 indicates that the CMR can be used as a unified evaluation index to quantitatively evaluate a structure's resistance against progressive collapse. For the original model, the CMR of the cylindrical latticed shell was 3.52 times greater than that of the spherical latticed shell, which had a CMR of 1.01. When the initial geometric imperfection was considered, the CMRs of the three latticed shell structures changed. The CMR of the cylindrical latticed shell increased by $35.5 \%$, while those of the other two latticed shells decreased. In particular, the CMR of the spherical latticed shell was less than 1. Thus, the structure's safety margin of resistance to progressive collapse was insufficient, and thus, there existed a severely higher risk of collapse after the failure of a single member.

The above analysis showed that the evaluation of progressive-collapse resistance based on IDA is not restricted by the structural form and that the structural performance in terms of resistance to collapse can be quantitatively evaluated. Moreover, the initial geometric imperfection should be considered; otherwise, incorrect or even dangerous evaluation results may be obtained.

\section{Engineering application}

\subsection{Project profile}

The roof of the main stadium for the Shenzhen Universiade Sports Centre has a single-layer folded-plane latticed shell structure system. The structural system is comprised of 20 units of similar shapes, and the dimensions of the architectural plane are $274 \mathrm{~m} \times 289 \mathrm{~m}$, as shown in Fig. 17. The sections of the main members are circular, and their diameters range from 700 to $1400 \mathrm{~mm}$. The materials used for the main members are Q390 and Q420.

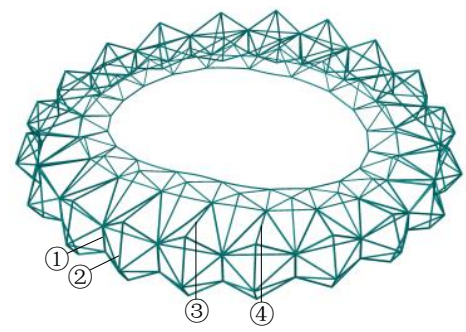

Fig. 17 Structural system of the main stadium

\subsection{Qualitative evaluation}

The important members were chosen from a quarter of the structure because of its symmetry using the multiple-response evaluation method based on the primary scope, which was employed by Tian et al. [7], as shown in Fig. 17. Decreasing the number of important members such that non-important members are eliminated is a conservative approach. Therefore, only four important members were evaluated.

Fig. 18 presents the DM-IM curves after the failure of each important member. The nonparametric method was used to calculate the $16 \%, 50 \%$, and $84 \%$ quantile curves for each DM, as shown in Fig. 19. First, the increase in the maximum displacement accelerated until a progressive collapse was finally triggered. At the collapse point, the displacement-span ratio was 1/30, and the combined load was greater than 1.2DL+0.5LL. Consequently, the main stadium for the Shenzhen Universiade Sports Centre was found to have a good resistance to progressive collapse. The entire structure has relatively good ductility. Second, the DM-IM curves of members 2 and 4 were identical, and thus, eliminating either of these two important members had a similar effect on the structure. However, because of the existence of member 1, the discreteness of the quantile curves increased under a large displacement. Third, the structure was significantly weakened by the failure of member 1 , which makes the importance of member 1 evident. Thus, threats and damage to member 1 should be avoided.

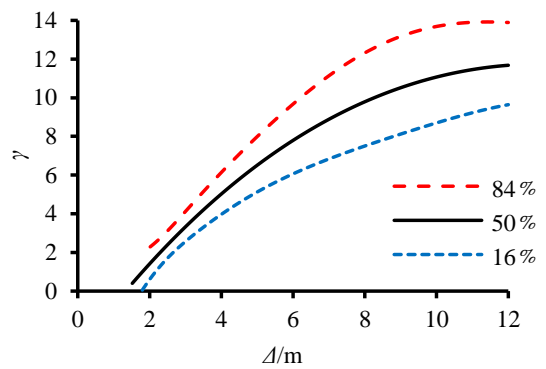

Fig. 19 Quantile curves for the project

\subsection{Quantitative evaluation}

Fig. 20 shows the vulnerability curves of the main stadium for the Shenzhen Universiade Sports Centre with a CMR of 1.68. According to the quantitative analysis, the main stadium showed good resistance to progressive collapse even though its cantilever length reached 51.9-68.4 m at some locations. The potential ability of the structure to resist progressive collapse was evaluated and expressed in the form of probability.

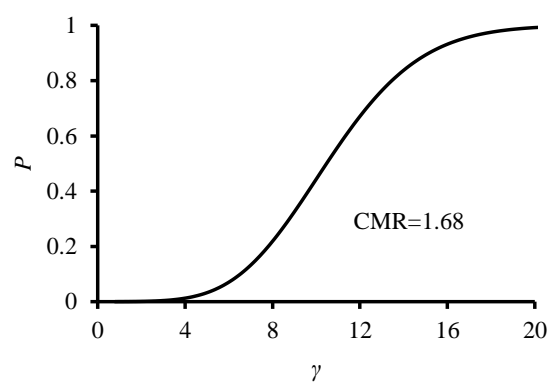

Fig. 20 Vulnerability curves for the project

Fig. 21 shows the collapse modes when the ultimate load condition is achieved. The deformation was concentrated in the structural unit that contained the failure member, and the fractures of the members were distributed around the failure member. This part of the structure is not appropriate for bearing the load, while the rest of the structural units are less affected by the failure member. Hence, dividing the structural units in the main stadium for the Shenzhen Universiade Sports Centre can effectively suppress the occurrence of progressive collapse. Three ring beams (i.e., bottom of the shoulder, bottom of the crown, and inner ring $[29,30])$ connect the entire structure, and the effect of them working together is apparent.

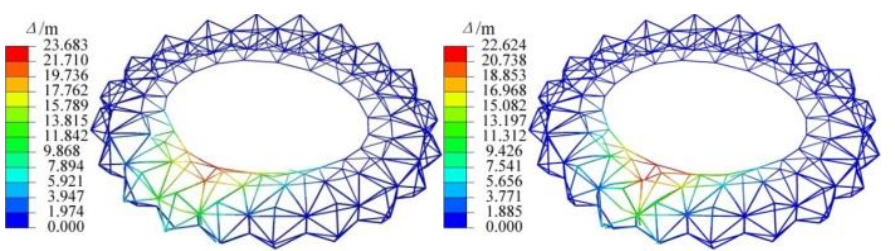

(a) Member 1 is removed

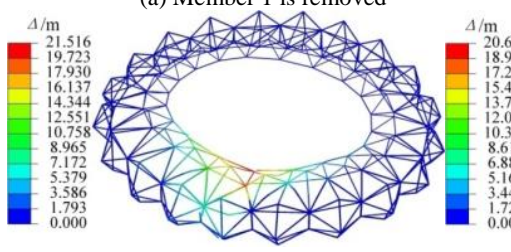

(b) Member 2 is remove

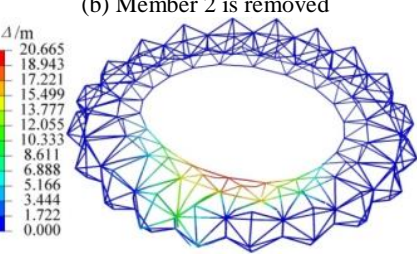

(d) Member 4 is removed

Fig. 21 Displacement distribution

\section{Conclusions}

A method of evaluating structural seismic performance known as IDA was intensively studied and used to develop a new method for evaluating the resistance to progressive collapse of long-span single-layer spatial grid structures. The proposed method was validated using three engineering case analyses and utilised in a computer simulation of the main stadium of the Shenzhen Universiade Sports Centre. The following conclusions were drawn:

(1) A suitable method for evaluating single-layer spatial grid structures called the evaluation of progressive-collapse resistance based on IDA was introduced. The method is not restricted by the structural form, and the method

Fig. 18 DM-IM curves for the project 
can quantitatively analyse the resistance of a structure to collapse.

(2) The displacement and strain responses can be predicted well with the FE model. In addition, the balance state that was regained matched the test observation. Thus, an efficient and accurate approach to studying the progressive collapse of single-layer spatial grid structures was developed.

(3) An initial geometric imperfection should be considered based on the consistent mode imperfection method; otherwise, incorrect or even dangerous evaluation results may be obtained.

(4) The main stadium for the Shenzhen Universiade Sports Centre was found to have good resistance to progressive collapse in the quantitative analysis even though the cantilever length reached 51.9-68.4 $\mathrm{m}$ at some locations. The potential ability of the structure to resist progressive collapse was evaluated and expressed in the form of probability.

\section{Acknowledgements}

This research was supported by the National Natural Science Foundation of China (Grant No. 51608433), the Science and Technology Co-ordination and Innovation Fund Project of Shaanxi Province of China (Grant No. 2016KTZDSF04-02-02), the Shaanxi Province Youth Science and Technology New Star Program (2018KJXX-20), the Shanghai Rising-Star Program (Grant No. 17QB1402300), and the Natural Science Foundation of Shaanxi Province of China (Grant No. 2018JQ5052). Their financial supports are greatly appreciated.

\section{References}

[1] ASCE 7-10, Minimum Design Loads for Buildings and Other Structures, American Society of Civil Engineers, 2010

[2] Rezvani F.H., Yousefi A.M. and Ronagh H.R., "Effect of span length on progressive collapse behaviour of steel moment resisting frames", Structures, 3, 81-89, 2015.

[3] Piroglu F. and Ozakgul K., "Partial collapses experienced for a steel space truss roof structure induced by ice ponds", Engineering Failure Analysis, 60, 155-165, 2016.

[4] Zhao X.Z., Yan S. and Chen Y.Y., "Comparison of progressive collapse resistance of single-layer latticed domes under different loadings", Journal of Constructional Steel Research, 129, 204-214, 2017.

[5] Biegus A. and Rykaluk K., "Collapse of Katowice fair building", Engineering Failure Analysis, 16, 1643-1654, 2009.

[6] Kamari Y.EI., Raphael W. and Chateauneuf A., "Reliability study and simulation of the progressive collapse of Roissy Charles de Gaulle Airport", Case Studies in Engineering Failure Analysis, 3, 88-95, 2015.

[7] Tian L.M., Wei J.P., Hao J.P. and Wang X.T., "Dynamic analysis method for progressive collapse of long-span spatial grid structures", Steel and Composite Structures, 23(4), 435444, 2017.

[8] Song B.I. and Sezen H., "Experimental and analytical progressive collapse assessment of a steel frame building", Engineering Structures, 56, 664-672, 2013.

[9] Sagiroglu S. and Sasani M., "Progressive collapse-resisting mechanisms of reinforced concrete structures and effects of initial damage locations", Journal of Structural Engineering, 140(3), 04013073, 2014.

[10] Yang B., Tan K.H., Xiong G. and Nie S.D., "Experimental study about composite frames under an internal column-removal scenario", Journal of Constructional Steel Research, 121, 341-351, 2016

[11] Demonceau J.F. and Jaspart J.P., "Experimental test simulating a column loss in a composite frame", Advanced Steel Construction, 6(3), 891-913, 2010.

[12] Yu H., Izzuddin B.A. and Zha X.X., "Progressive collapse of steel-framed buildings: influence of modelling approach", Advanced Steel Construction, 6(4), 932-948, 2010.

[13] GSA 2013, Progressive Collapse Analysis and Design Guidelines for New Federal Office Buildings and Major Modernization Projects, Washington DC: General Services Administration, 2013.

[14] UFC 4-023-03, Design of Buildings to Resist Progressive Collapse, Washington DC: Department of Defense, 2013

[15] CECS 392, Code for Anti-collapse Design of Building Structures, China Association for Engineering Construction Standardization, 2015.

[16] Malla R.B., Agarwal P. and Ahmad R., "Dynamic analysis methodology for progressive failure of truss structures considering inelastic postbuckling cyclic member behavior", Engineering Structures, 33, 1503-1513, 2011.

[17] Thai H.T. and Kim S.E., "Nonlinear inelastic time-history analysis of truss structures", Journal of Constructional Steel Research, 67, 1966-1972, 2011

[18] Jiang X.F. and Chen Y.Y., "Progressive collapse analysis and safety assessment method for steel truss roof", Journal of Performance of Constructed Facilities, 26(3), 230-240, 2012.

[19] Liu S.W., Bai R., Chan S.L. and Liu. Y.P., "Second-order direct analysis of domelike structures consisting of tapered members with I-sections", Journal of Structural Engineering, 142(5), 04016009, 2016

[20] Liu Y.P., Chan S.L., Du Z.L. and He J.W., "Second-order direct analysis of long-span roof structures", The 8th European Conference on Steel and Composite Structures, 1(2\&3), 3930-3939, 2017

[21] Fascetti A., Kunnath S.K. and Nisticò N., "Robustness evaluation of RC frame buildings to progressive collapse", Engineering Structures, 86, 242-249, 2015

[22] Vamvatsikos D. and Cornell C.A., "Incremental dynamic analysis", Earthquake Engineering and Structural Dynamics, 31, 491-514, 2002.

[23] Zhou Y., Lv X.L. and Bo Y., "Application of incremental dynamic analysis to seismic evaluation of hybrid structure", Journal of Tongji University (natural science), 38(2), 183187, 2010. [in Chinese]

[24] Shi W., Ye L.P., Lu X.Z and Tang D.Y. "Study on the collapse-resistant capacity of RC frames with different seismic fortification levels", Engineering Mechanics, 28(3), 41-48, 2011. [in Chinese].

[25] Yu X.H. and Lv D.G., "Seismic collapse fragility analysis considering structural uncertainties", Journal of Building Structures, 33(10), 8-14, 2012.

[26] FEMA-350, Recommended Seismic Design Criteria for New Steel Moment-frame Buildings, Washington D. C: Federal Emergency Management Agency, 2000.

[27] Wang W.M., Li H.N. and Tian L., "Progressive collapse analysis of transmission tower- line system under earthquake", Advanced Steel Construction, 9(2), 161-172, 2013.

[28] JGJ 7-2010, Technical Specification for Space Frame Structures, Ministry of Housing and Urban-Rural Development of the People's Republic of China, 2010.

[29] Tian L.M. and Hao J.P., "Nonlinear time-varying analysis algorithms for modeling the behavior of complex rigid long-span steel structures during construction processes", Steel and Composite Structures, 18(5), 1197-1214, 2015

[30] Tian L.M., Wei J.P., Hao J.P., "Anti-progressive collapse mechanism of long-span singlelayer spatial grid structures”, Journal of Constructional Steel Research, 144, 270-282, 2018. 